\section{Rehabilitación post COVID-19: un desafío vigente}

\section{Post COVID-19 rehabilitation: A current challenge}

\section{Señor Editor,}

Es indudable que la pandemia por COVID 19 nos ha puesto a prueba. La actual crisis sanitaria ha obligado a los sistemas de salud del mundo a redoblar esfuerzos para evitar el colapso del sistema sanitario debido a la alta demanda de camas de distinta complejidad, necesarias para intentar mantener con vida a quienes se han visto más severamente afectados. Esto, sin embargo, a expensas de largos periodos de aislamiento en terapia intensiva y el consiguiente deterioro funcional, manifestado en alteraciones psicológicas, cardiorrespiratorias y musculo-esqueléticas, cuyas secuelas tienen un impacto directo en el pronóstico funcional de estos pacientes ${ }^{1}$.

Nuestro país no ha estado ajeno a esta realidad.
A la fecha de la redacción de esta carta, reportes del Ministerio de Salud muestran cifras superiores a 430 mil casos acumulados, de los cuales más de 34 mil han debido ser hospitalizados ${ }^{2}$. De estos, $68,9 \%$ tiene más de 50 años y en su inmensa mayoría, ademas cursan con enfermedades crónicas no transmisibles de alta prevalencia en nuestro pais. Esto último nos plantea enormes desafíos en el seguimiento y la rehabilitación de quienes logran sortear la enfermedad. Informes recientes indican que luego de dos meses del inicio sintomático del cuadro clínico, $87,4 \%$ persiste con a lo menos 1 síntoma y $55 \%$ con a lo menos 3 , prevaleciendo la fatiga $(53,1 \%)$, la disnea $(43,4 \%)$, el dolor articular $(27,3 \%)$ y el dolor torácico $(21,7 \%)^{3}$.

Este fenómeno impacta no solo al individuo, sino también a su familia directa y a toda la comunidad. Se ha observado que un tercio de quienes se encuentran en edad laboral y experimentan Sindrome post UCI luego de sufrir Sindrome de Distress Respiratorio Agudo no 
vuelve a trabajar y otro tercio no regresa a su trabajo previo o no vuelve a recibir una remuneración similar a la inmediatamente anterior a la de su paso por cuidados críticos. Además, al menos $25 \%$ de quienes fueron sometidos a terapia intensiva experimenta una disminución dramática de su independecia posterior al año de recibida el alta médica, lo que supone un detrimento en la calidad de vida del paciente y un aumento del gasto en salud de las familias, asociado a la carga derivada por los cuidados de salud formales o informales 4 .

Como respuesta, diversas sociedades científicas han puesto en evidencia la necesidad implementar programas de rehabilitación desde la fase aguda de la enfermedad, con especial énfasis en pacientes que han requerido ventilación mecánica, en quienes cuenten con enfermedades pre-existentes y en aquellos con edad avanzada ${ }^{5}$. En este escenario, los profesionales de la rehabilitación adquieren un rol protagónico al favorecer la restauración de la funcionalidad y la independencia en actividades cotidianas, mediante la prescripción de ejercicios y protocolos de intervención acorde de las necesidades propias de cada inidividuo.

En este contexto, la red de Atención Primaria de Salud (APS) distribuida a lo largo de todo Chile, tiene la oportunidad y el deber de tomar un rol protagónico en esta materia. Su instalación a nivel comunitario y su enfoque de salud familiar, junto a un equipo de salud comprometido y capacitado en este modelo, ofrecen la oportunidad de proyectar los objetivos de la rehabilitación hacia los contextos reales de las personas, dando continuidad a la cadena de rehabilitación que se inicia desde las unidades de cuidados intensivos y que debe desembocar necesariamente en una adecuada reinserción social. Lamentablemente, si no se llega a este eslabón final, gran parte de los esfuerzos realizados en etapas previas pierden sentido.

Junto a lo anterior, la APS ofrece prestaciones relevantes para el contexto clínico de estos pacientes, involucrandose en un trabajo directo con las familias y sus cuidadores/as quienes frecuentemente se ven obligados a asumir nuevos roles asociados al apoyo que deben brindar en el proceso de rehabilitación con el consecuente impacto en su salud. Esta red cuenta además con equipos de salud rural que pueden realizar atenciones domiciliarias en sectores de difícil acceso de nuestro país, mejorando la equidad en la cobertura de estas prestaciones. En este sentido, la estrategia de Rehabilitación de Base Comunitaria (RBC) cobra relevancia, al ofrecer respuestas a las necesidades actuales de nuestra sociedad derivadas de esta pandemia en base a estrategias de desarrollo comunitario, igualdad de oportunidades e inclusión social de personas con discapacidad, la que sin duda debe ser fortalecida en pos de potenciar la participación del usuario, su familia y las comunidades, fomentando la co-construcción del proceso de rehabilitación para llegar a la meta final: el logro de la inclusión o reinserción social, en cualquiera de sus áreas (familiar, educativa, laboral o comunitaria $)^{6}$.

Frente a esta nueva realidad, será imperativo cubrir las necesidades asistenciales y de reinserción social asociadas a la rehabilitación integral de este tipo de pacientes, como de todos aquellos que han visto postergadas sus atenciones regulares involucrando niños, niñas, adolescentes, adultos y personas mayores. La urgencia en el acceso y oportunidad de atención, impactará de manera sustancial en su calidad de vida, de la de sus familias y el adecuado funcionamiento de la sociedad en general.

Roberto Acosta-Dighero ${ }^{1,2, a}$, Iván RodríguezNúñez ${ }^{2,3, a, b}$, María José Solís-Grant ${ }^{2,3, a}$, Rodrigo Torres-Castro ${ }^{4,5, a}$, Carolina García-Soto ${ }^{1}$ ${ }^{1}$ Unidad de Rehabilitación con Atención Clínica Integral (URACI), Hospital Roberto del Río, Santiago, Chile. ${ }^{2}$ Fundación Kinesiología, Ciencia y Sociedad. ${ }^{3}$ Departamento de Kinesiología, Facultad de Medicina, Universidad de Concepción. Concepción, Chile. ${ }^{4}$ Departamento de Kinesiología, Facultad de Medicina, Universidad de Chile, Santiago, Chile. ${ }^{5}$ International Physiotherapy Research Network (PhysioEvidence). ${ }^{a}$ Kinesiólogo $(a)$. ${ }^{b}$ Doctor en Ciencias Médicas.

\section{Referencias}

1. Candan SA, Elibol N, Abdullahi A. Consideration of prevention and management of long-term consequences of post-acute respiratory distress syndrome in patients with COVID-19. Physiother Theory Pract 2020; 36 (6): 663-8.

2. MINSAL. Informe Epidemiológico $n^{\circ}$ 43. Enfermedad por SARS-CoV-2 (COVID-19). In: Epidemiología, editor. Gobierno de Chile ed2020.

3. Carfi A, Bernabei R, Landi F, Group ftGAC-P-ACS. Persistent Symptoms in Patients After Acute COVID-19. JAMA 2020; 324 (6): 603-5.

4. Stam HJ, Stucki G, Bickenbach J. Covid-19 and Post Intensive Care Syndrome: A Call for Action. J Rehabil Med. 2020; 52 (4): jrm00044.

5. Consenso Interdisciplinario de Rehabilitación Post COVID-19. (2020) https://sochimfyr.cl/site/docs/Consenso_20_de\%20Agosto.pdf

6. Solís MJ, Quezada R. Sistema de rehabilitación en la red asistencial. En M. Tamayo, A. Besoain-Saldaña, y J. Rebolledo. (Eds.), Kinesiología y discapacidad: Perspectivas para una práctica basada en derechos (pp. 32-49). Santiago, Chile. Universidad de Chile. 2020.

Correspondencia a:

Roberto Acosta-Dighero

Unidad de Rehabilitación con Atención Clínica Integral (URACI),

Hospital Roberto del Río. Zañartu 1085, Independencia. Santiago, Chile.

acostadighero@gmail.com 